\title{
Camille - Reaktivierter Konflikt, Übertragung und Wunsch-Abwehr-Dynamik
}

\author{
Dominic Suter (Zürich)
}

Zusammenfassung: Camille, eine Frau Mitte dreissig, ist in einer Beziehung mit einer Frau und wünscht sich ein Kind. Im Lauf der Analyse arbeiten wir uns zu ihrem Wunsch vor: eine gleichberechtigte, ebenbürtige Beziehung mit einem Mann. Doch jedes Mal, wenn sie ihren Liebeswunsch an einen Mann richtet, gerät sie in Angst und blockt ab. Ihre Einfälle haben hauptsächlich zum Inhalt, dass etwas kaputt oder verlustig gehen könnte.

Schlüsselwörter: Triebtheorie, Ödipuskomplex, passiv-genitaler Wunsch, Kastrationstat, Kastrationsangst

Im Zentrum der analytischen Arbeit steht meines Erachtens das Erforschen der Triebdynamik zwischen dem Wunsch und der mit ihm verbundenen Angst, die sofort abgewehrt wird. Den verpönten unbewussten Wunsch, den «Willen» und die mit ihm aufkommende «Angst», den «Gegenwillen» (Freud, 1900, S. 333), zu erkennen, ist nicht immer einfach. Zu Beginn einer Therapie, wenn meine Aufgabe darin besteht, von der äusseren Welt zur seelischen Realität im Innern zu lenken, bewährt es sich, nicht beim Wunsch, sondern, ganz nach Freud, beim Hindernis anzusetzen: dem Gewissen und der Abwehr der Angst. ${ }^{1}$ Die Arbeit von aussen nach innen ist die Arbeit am Widerstand (vgl. Freud, 1895d).

Um die Analysandin darin zu begleiten und zu unterstützen, immer tiefer in ihr Inneres vorzustossen und die Elemente ihrer Wunschvorstellung und ihrer jeweils aufkommenden Angstvorstellung zu erkennen und zu verstehen, achte ich nicht zuletzt darauf, was sie in welcher Abfolge sagt, und spreche diese Sukzession (vgl. Morgenthaler, 2005) auch explizit an. Dabei arbeite ich mit der Theorie von Judith Le Soldat, die Freuds Gedankengebäude, insbesondere dessen ödipale Theorie, entscheidend weiterentwickelt hat (Le Soldat, 2015, 2020; vgl. auch Fäh, 2018, 2021) - zum einen mit innovativen Antworten auf die Frage «Was will das ödipale Kind?», zum andern mit dem Kerngedanken, der Untergang des Ödipuskomplexes sei in der Kastration des Vaters begründet. Wie Le Soldat stringent argumentiert, geht der Angst vor dem Vater die im Innern aktiv ausgeführte 
Kastrationstat voraus. Aus diesem Verständnis lassen sich zahlreiche neurotische Phänomene präzise erklären. Am Fall Camille, den ich in der Supervision regelmässig mit Elisabeth Geiger besprochen habe, lässt sich beispielhaft aufzeigen, welch grosse Hilfe Le Soldats erweiterte ödipale Theorie in der praktischen Anwendung sein kann, um auf die innere Bühne der Analysandin oder des Analysanden zu gelangen.

Camille, eine Frau Mitte dreissig, kommt seit einem Jahr in die Analyse. Sie liegt. Zu Beginn ist sie überzeugt, sie wolle mit Hannah, der Frau, mit der sie in einer Beziehung ist, ein Kind - via Samenspende; gleichzeitig denkt sie, dass sich Kind und Karriere eigentlich ausschliessen. Schon bald zeigt sich aber, dass es doch einen Mann gibt, von dem sie mehr will: Nennen wir ihn Gustave. Aber da gibt es ein Hindernis.

\section{$1 \quad$ Zu teuer}

Als erste Folge der begonnenen Behandlung erwägt Camille, Gustave zu besuchen. «Ich wäre gern zu ihm nach Frankreich gefahren», sagt sie. «Doch ich weiss ja nichts von seinem Leben. Ich stand schon auf dem Perron, aber das Zugticket war zu teuer, ich entschied mich dagegen.» Die Abfolge des Gesagten gibt erste Hinweise auf ihre innere Position. Ihre Zuwendung zu einem Mann stellt sich in ihrer Innenwelt als «zu teuer» dar: Sie befürchtet, zahlen zu müssen, zur Kasse gebeten zu werden. Nachdem ich ihr diese Angst gedeutet habe, fasst sie einen Besuch doch wieder ins Auge. Doch dann sendet ihr Gustave per WhatsApp ein Bild seines erhobenen Fingers - manifest, um ihr zu zeigen, dass sein Finger zwar noch leicht blute, aber am Verheilen sei. Erneut kühlt Camilles Stimmung ab, erneut zögert sie, ihn zu besuchen. Weshalb? Betrachtet man die Abfolge, ist es der blutende Finger, der sie zögern lässt - der ihr Angst macht. Ich deute ihr, dass sie den erhobenen Finger offenbar als Mahnung und Vorwurf interpretiert. Offen bleibt vorläufig, was das Blut bedeutet.

\section{Die Hand ausstrecken - die Hand nehmen}

«Mein Kollege Paul erzählte mal von einem Übergriff», erwähnt Camille daraufhin:

In einer Nacht schliefen Paul und Pamela zusammen. Pamela war im Halbschlaf, er checkte es nicht. Nun gibt es deshalb mit den beiden eine mega komplizierte Konstellation [im Hinblick darauf], wer wo eingeladen werden darf. 
Ich: «Übergriff: ein interessantes Wort.»

Sie: «Der Ekel. Mein Kollege David legte mir kürzlich die Hand auf den Bauch. Too much! Auf den Bauch! Das macht man nur bei Schwangeren. Ich bin am Bauch auch im Yoga so empfindlich. Selbst als ich mit vermeintlich besten Freunden im Bett geschlafen habe: Da musste ich am Morgen eine Hand wegtun! So ein Schreckmoment! Nicht nur freundschaftlich, noch was anderes.»

Ich: "Es graut Ihnen davor. Im Morgengrauen kommt etwas Feindliches, das Gegenteil von freundschaftlich. Sie geben sich hin, schlafen ein und, zack, langt der andere rüber. Grausig.»

Sie: «Es hat so etwas Besitzergreifendes.»

Gustaves «Mahnfinger» weckt bei ihr also die Erinnerung an einen «Übergriff» im Schlaf. Ich mache mir folgende Gedanken dazu. Die Hand greift nach dem Körper: Sie will etwas nehmen, wegnehmen. Damit kommt das Thema des Hinübergreifens, das ein Inbesitznehmen ist, zur Sprache. Wenn eine Person dermassen Angst hat, ihr könnte etwas genommen werden, kann dies gemäss dem im Psychischen herrschenden Talionsprinzip ${ }^{2}$ ein Hinweis darauf sein, dass sie ebendies selbst getan hat. Wenig später in dieser Stunde ergänzt Camille:

Letzte Nacht träumte ich. Im Traum kommen Sie vor. Wir fuhren im Auto. Sie fuhren, ich streckte die Hand aus, habe sie offengelegt, und Sie nahmen meine Hand.

Wie Camille zuvor erzählt hat, hat sie Angst, wenn sich eine Hand nähert. Nun berichtet sie vom «Autofahren», was ich als Hinweis auf den Sexualverkehr lese. Camille hat die Hand ausgestreckt, offen hingelegt. Das innige «wir» ist ein Zeichen dafür, dass sich die ödipale Vaterübertragung etabliert hat. Camilles passiv-genitaler Wunsch zeigt sich deutlich: Sie möchte gefahren werden, doch die Gefahr besteht, dass der andere nimmt. Camille fährt fort:

Mit Gustave habe ich hinten im Auto jeweils heimlich Händchen gehalten. Das war ein mega Ding. Alles gut und schön, bin mega verliebt, alles intuitiv und sehr schön. Gustave ist aktuell ja voll in der Krise. Sonst macht er immer, was er fühlt. Er sagte, er habe das Traumhaus in Frankreich gefunden, müsse es kaufen, könne sich aber nicht überwinden, weil er einen millionenschweren 
Fehler begehen könnte. Er zeigte es mir. Ich fand, es sehe aus wie ein Gefängnis. Ich schrieb ihm: «Dann muss ich loslassen von der Vorstellung, [wir] zusammen in der Schweiz.» Er sagte, das grenze an Utopie. Ich weinte. Utopie heisst: eine schöne Vorstellung, aber auch weit weg.

In den Assoziationen «mega verliebt» und «sehr schön» drückt sich meines Erachtens das innige Bild ihres passiv-genitalen Wunsches aus. Aber «die Hand ausstrecken» beinhaltet auch, dass ihr etwas weggenommen werden kann. «Sonst macht er immer, was er fühlt», weil man jedoch für einen «Fehler» «teuer» bezahlen könnte, weil gar «Gefängnis» droht, macht man nicht mehr, was man fühlt. Um der Strafe zu entgehen, glaubt Camille, nicht nur ihre Idee vom Haus, sondern auch vom harmonischen, gemeinsamen Leben als liebendes Paar loslassen zu müssen.

In der Übertragung richtet Camille den passiv-genitalen Wunsch nun an mich. Meine Gegenübertragung spricht aber nicht dafür, dass in ihrem Traum das Libidinöse obsiegt: So meldet sich bei mir unmittelbar die Vorstellung, sie greife nach dem «Schaltknüppel», also nach meinem Phallus, deutlich spüre ich ihre Angst, die nun im Raum steht. Da ich vermute, dass diese Angst der Grund dafür ist, dass sie ihren Traumbericht unterbrochen hat, möchte ich von ihr wissen:

Ich: «Was war sonst noch im Traum? An welcher Stelle sind Sie aufgewacht?»

Sie: «Der Traum ging weiter, es ging den Hang hinunter, wir sind beinahe aus der Kurve gefallen. Und das Lieblingslied vor zehn Jahren: die CD, die Sie reintaten, Musik von früher, der Phase von zwanzig bis fünfundzwanzig. Meine Kollegin sagte, wenn Gustave und ich zusammen seien, fühle man sich ausgeschlossen, weil wir schon mega gut zusammenpassen. Für meine Freundin Hannah ist das halt eine mega Bedrohung.»

Ich: «Hier sind zwei Vorstellungen mit der Hand verbunden: Es ist zwar schön und innig - aber nicht auch gefährlich?»

Sie: «Es ist schon was anderes, wenn ich die Hand nach vorn gebe.»

Eine enorme Angst taucht auf («den Hang hinunter», «beinahe aus der Kurve gefallen»), deren Wucht ich meiner Gegenübertragung entnehme und die sich mehrfach bestätigt: in Camilles Einfällen («Abgrund, wo das Auto dann hinzufahren droht»), in Gustaves Krise, der Gefängnis-Assoziation zum Haus und der «mega 
Bedrohung» für ihre Freundin Hannah, die immer wieder als ihr Alter Ego fungiert. Ein Lied von früher wird Thema. Sogar Aussenstehende sehen, wie verliebt Camille und Gustave sind («mega gut zusammenpassen»).

Fassen wir einmal mehr zusammen: Sobald Camille ihren passiv-genitalen Wunsch zulässt, stellt sich etwas «dazwischen». Ihr kommt ein Gefängnis in den Sinn, damit verbunden das Motiv «zu teuer»: IhrWunsch könnte sie zu viel kosten. Zudem kommt die Angst auf, jemand könnte feindselig nach ihr «greifen». Da ihr also eine Strafe droht, besteht Camilles sofort mobilisierte Angstabwehr darin, ihre innige Vorstellung «loszulassen», wodurch ihre Wunscherfüllung wieder in die Ferne rückt («Utopie»). Ihr Wunsch ist demnach weniger für Hannah eine «mega Bedrohung» als vielmehr für Camille selbst.

Camilles Angst, die im Zentrum wirkt, spüre ich, wie erwähnt, deutlich. Doch weshalb glaubt sie, ins Gefängnis zu müssen, wenn sie sich ihren Wunsch erfüllen will? Und woher rührt diese heftige Angst, ihr werde etwas genommen? Daher, dass sie bei aller «Verliebtheit» selbst schnell ans «Zugreifen» denkt? Welche «Erinnerung» ist damit verknüpft?

Ich: «Was ist Ihnen so peinlich bei dieser Vorstellung im Auto?» Sie: «Intim ist es. Es ist Übertragung, klar. Aber dennoch. Es kostete Überwindung, das zu erzählen. Es ist schon peinlich, weil Sie es sind. Es war mega aufgeladen. An mehr kann ich mich nicht erinnern.»

Ich: «Welches Lied?»

Sie: «Eine CD, nostalgisch. [Camille nennt einen Songtitel, in dem ein Löwe vorkommt].»

Ich: «Löwe. Was kommt Ihnen zum Wort Löwe in den Sinn?»

Sie: «Raubkatze. Es ist auch Gustaves Sternzeichen. Ich war mit Hannah ein Wochenende bei meinen Eltern, es tat so gut, war aber komisch, mit einer Frau dort zu sein.»

Bemerkenswert ist, dass Camille, sobald das Gespräch zur Raubkatze führt, auf Gustave verweist, als müsste sie von etwas ablenken: vom Gefängnis, einer hohen Geldsumme, einem Raub? Die Raubkatze ist mit Camilles altem Lieblingslied verknüpft: Könnte man daraus schliessen, dass Rauben eine alte Lieblingsbetätigung ist? Mit dem Element Raub scheint sich uns nun die Bedeutung ihres Traumes zu erschliessen. Camilles federführender Wunsch ist, «gefahren zu werden», sich «auszustrecken»-also der passiv-genitale Wunsch. Die Traumerzählung wird dort 
unterbrochen, wo ich ihre Hand «nehme»: Sofort entwickelt Camille Angst. Die Hand nehmen kann doppelt gedeutet werden: als Liebe, aber auch als Raub.

Dass Camille als letztes Element «mit einer Frau dort sein» einbringt, ist im Hinblick auf die Abfolge ihrer Einfälle wichtig. Wenn die Raubkatze auftaucht, schiebt Camille die Schuld auf Gustave; sobald sie ihren Impuls zu rauben auf ihn projiziert, steigt ihre Angst, er werde sie berauben. Am Ende dieser Sukzession ist sie «bei einer Frau»: Wenn Camille Angst vor dem Mann bekommt, wechselt sie zur Frau. Ich sage zu Camille:

Gefahren werden, sich ausstrecken können, das wäre schön. Sie möchten lieber von einem Mann gefahren werden, aber Sie haben eine Riesenangst, Ihre Autonomie gerate aus der Bahn, es «koste» etwas: Abgrund, Krise, Gefängnis, eine mega Bedrohung!

Damit mache ich eine klassische Konfliktdeutung. Ich spreche Camilles Wunsch und Angst an und zeige ihr auf, dass ihre Angst so gross ist, dass sie bereit ist, ihren Wunsch fallen zu lassen.

In der Folgestunde herrscht eine andere Stimmung. Camille berichtet, sie habe am Telefon «banal und beliebig» mit Gustave gesprochen. Nachdem sie ihren Wunsch heftig an Gustave und mich gerichtet hatte, hat nun offenbar ihre Angst, etwas hergeben zu müssen, wieder die Oberhand gewonnen. Die Verharmlosung ihrer Beziehung zu Gustave lässt sich als Rückzieher zur Abwehr dieser Angst interpretieren. Somit ist Camilles Wunsch vom Tisch.

\section{Der rasierte Penis}

Zwei Wochen später berichtet Camille etwas Überraschendes:

Gestern Sonntag wurde ich abgeschleppt. [...] Ich war null vorbereitet. Er hat mega gestürmt, er ging grad rein. Mit ihm Sex und sonst nichts, sagte er. Ich wollte es auch mal hinter mich bringen.

Camille hatte also Sex mit einem Mann. Es ist längere Zeit her seit ihrer letzten sexuellen Begegnung mit einem Mann, da sie diesbezüglich eine starke Hemmung hat. Weshalb ist dies nun plötzlich möglich? Camille erzählt, wie es dazu gekommen ist: 
Am Tag davor hatte ich einen komischen Chat mit einem, der Sex wollte. Der ist mega attraktiv, aber ein Idiot. Er schickte mir auf WhatsApp ein Bild seines Schwanzes, und der war rasiert; er sei nicht interessiert, wenn ich nicht rasiert bin für ihn. Ganz rasiert mache ich nie, ich hab ein Problem mit diesem Meitli-Ding, das stört mich. Danach hyperventilierte ich.

Camille erhält also das Bild eines erigierten Penis, doch als Erstes fällt ihr auf, dass etwas fehlt. Darauf reagiert sie mit «rasiert mache ich nie!», sie will um jeden Preis verhindern, im Gegenzug etwas Wertvolles hergeben zu müssen - ihr (Scham-)Haar ${ }^{3}$. Ihr anschliessendes Hyperventilieren interpretiere ich als Hinweis darauf, dass dieses Bild massive Angst auslöst - die sie aber nicht also solche wahrnimmt.

Das Ziel dieser Analyse ist meines Erachtens, dass Camille die enorme Angst spürt, die sie erfasst, sobald sie einen Schritt auf einen Mann zu macht. Technisch geht es hier darum, mit ihr herauszuarbeiten, was am Samstag und was am Sonntag geschah, um herauszufinden, worin genau die Bedrohung besteht, wenn ein Mann ihr nahekommt.

Aus Camilles Erzählung ziehe ich folgendes Fazit. Ein Penis und ein stattgefundener (Haar-)Schnitt «erinnern» sie an Gustaves blutigen Mahnfinger (siehe S. 123). Sie sieht darin vonseiten des Mannes einen Vorwurf («ich bin rasiert!»), aber auch eine Drohung («auch du wirst rasiert, du wirst etwas hergeben müssen!»), die sie in ihrem Innern bereits als unabwendbare Tatsache erlebt: Am Samstag droht ihr eine Rasur. Am Sonntag überwindet sie ihre Angst jedoch unversehens - ein Mann muss her, einer, der Gas gibt, und zwar sofort.

Aus Angst, ein Mann könnte etwas Ungeheuerliches (eine Rasur) von ihr fordern, seine Hand könnte ihr an den Bauch greifen, hatte sie eineinhalb Jahre lang keinen Sex. Camilles plötzliche Wahl eines draufgängerischen Mannes erfolgt nun also nicht aufgrund ihres passiv-genitalen Wunsches, sondern aus purer Angstabwehr, nach dem Motto «Lieber ein Ende mit Schrecken als ein Schrecken ohne Ende». ${ }^{4}$ Sie verhält sich kontraphobisch in der Hoffnung, auf diese Weise ihre Angst loszuwerden, die in ihrem Innern wirkende Angst vor der Rasur; diese Angst, dass ein Mann ihr «etwas wegnehmen» könnte, ist es, die Camille daran hindert, sich liebevoll an ein potentes männliches Gegenüber zu wenden.

Doch da sie selbst diese Angst nicht spürt, muss ich sie ihr deuten. Daher sage ich zu ihr: «Der [Mann] vom Samstag will, dass Sie etwas hergeben, abschneiden.» Ein rasierter Mann fordert, nach dem Talionsprinzip, auch von ihr eine Rasur. 
Dies löst eine neue Angstabwehr aus, eine kontraphobische Flucht nach vorn: Sie will es «hinter sich bringen». Diese Abfolge zeige ich Camille auf, um ihr zu ermöglichen, den eigentlich federführenden, liebevollen Wunsch wiederaufzunehmen und ihre Angst dabei zu ertragen.

Psychoanalytisch interessant ist übrigens Camilles Formulierung «ein Bild seines Schwanzes, und der war rasiert». Damit meint sie vermeintlich bloss, dass die Schamhaare wegrasiert sind, in der Verkürzung entsteht daraus jedoch ein neues Bild: der (weg-)rasierte Penis.

\section{4 «Geschichten, die schon in mir drin sind»}

Drei Wochen später fährt Camille nach Frankreich und besucht Gustave. Danach erzählt sie:

Ich vergesse oft, wie enttäuscht ich von ihm bin, zwar «krass angezogen», manchmal fand ich ihn aber «so wüst». Er ist schon sehr gross, und ich werde zum kleinen Meitli. Dann wollten wir Sex. Und wir kamen so in ein Zeug. Wir begannen und hörten wieder auf. Es war gut. Ich hatte es halb unter Kontrolle. Minimstes kann auslösen: Nein, es geht nicht. Eine falsche Bewegung, und es verpufft. Ich: "Was war die falsche Bewegung, worauf es verpuffte?»

Sie: «Er sagte: «Du bist so fein, du musst aggressiver sein〉. Er hätte es gebraucht, damit es mehr reisst. Ich hatte es so unter Kontrolle. So habe ich ihn im Griff.»

Ich: «Weshalb ihn kontrollieren? Gefährlich, wenn er was macht?» Sie: «Ich wünschte es mir ja so, hatte aber so Angst. Es ist ja mega aufgeladen mit ihm, es passte so gut sexuell. Ich hatte mega Angst. Ich hatte mega Lust. Wenn er aber bremst, bremse ich auch. [...] Bei Gustave im Bett schwitzte ich mega. Es war die Angst! Und eiskalte Hände und Füsse hatte ich. Ich habe das Gefuul [statt ‘Gefühl〉 macht sie den Versprecher ‘Gefuul, also ‘Gefaule〉], dass unsere Geschichte bereits angefault ist. Er roch meine Mens. Ich dachte, er riecht etwas.»

Ich: "Etwas von der faulen Geschichte kommt raus.»

Sie: «Geschichten, die schon in mir drin sind. Obwohl meine Kollegin mit mehr Männern was hatte als ich.» Ich: "Es kann also eine andere Geschichte gemeint sein.» 
Sie: «Es hat mit meinem Charakter zu tun und mit Entscheidungen, wie ich sie im Leben getroffen habe. Er hat nicht gern, wenn ich so zögerlich, zu lieb und sanft bin.»

Was fällt hier auf? Camille beschreibt eine «krasse» Anziehung gegenüber Gustave, worauf er unmittelbar als feindliche Figur («so wüst») aufscheint. Zur Abwehr ihrer Angst macht sie sich klein, idealisiert den Mann («Er ist schon sehr gross, und ich werde zum kleinen Meitli»), wird vorsichtig; sie glaubt, etwas im Griff behalten, vor dem Zugriff bewahren zu müssen. Camille und Gustave erleben grösste Anziehung und grösste Angst, beide wollen, aber bei beiden wird eine Art Bremse aktiviert: Es stellt sich etwas zwischen sie. Für Camille ist es ein Warnsignal, wenn Gustave ganz besonders will - und ebenso ein Alarmsignal, wenn er nicht so stark will. ${ }^{5}$ Nie weiss sie: Wird er plötzlich feindlich? In dieser Analysestunde zeigt sich, dass Camille sowohl Lust als auch Angst spürt - und dass sie nicht ausweicht. Dafür lobe ich sie.

Ich: «Sie sind in die Höhle des Löwen gegangen. Sie spüren die Angst sehr gut: den Schweiss, die eiskalten Hände. [...] Was ist das Gegenteil von sanft?»

Sie: «Aggressiv, impulsiv.»

Ich: «Offenbar waren Sie bei jener Entscheidung nicht zögerlich und sanft, jener Entscheidung, die charakterbildend war. Die Vorsicht und Angst könnten mit etwas zusammenhängen, wo Sie nicht sanft waren. Es gibt eine Hemmung: Wenn man richtig will, dann kommt die Anklage.»

Im analytischen Einkreisen stossen wir auf Camilles Angst, dass «eine Geschichte» auskommen könnte. Diese Formulierung klingt, als drohe ihr, dafür vor Gericht zu kommen und verurteilt zu werden. Die Anklage lautet: zu aggressiv, zu impulsiv! Welche «Geschichte» auf ihrer inneren Bühne ist damit gemeint? Dass sie sich Gustave gegenüber sanft zeigte, lese ich als eine Reaktionsbildung auf und gegen ihren Raubkatzen-Impuls.

In der darauffolgenden Stunde beschäftigt sich Camille wieder mehr mit Frauen. Ihre Annäherung an Gustave und mich hat ihre Angst hochschnellen lassen; diese Angst lässt sie vor den Männern erneut wegrennen. Zur Frau zu gehen, war bis jetzt ihre bewährte Angstabwehr. Doch nun taucht ein ungewohntes Ele- 
ment auf: Voller Verzweiflung sagt Camille, sie sei «ruiniert». Hier zeigt sich eine neue Art von Angstabwehr: Sie identifiziert sich mit einer kaputten Figur. ${ }^{6}$

Wovor hat Camille im Innern Angst? Meines Erachtens können wir es nun wagen, die gesammelten Erkenntnisse zu einer Antwort zusammenzufügen. Camilles starke Anziehung zu einem Mann weckt stets starke Angst, gar Panik. Wie sich gezeigt hat, hat ihre Angst etwas mit einem «blutenden Finger», einem «Übergriff», mit «die Hand nehmen», einer «Rasur», einem «rasierten Penis», mit «Haaren», die mit Potenz verbunden sind, einem «Raub», einem «Raubtier», mit dem Gefühl, «zu aggressiv, zu impulsiv» zu sein, und mit einer «ruinierten» Figur zu tun.

All diese Elemente evozieren das Bild einer Kastration.

Wie Freud erkannte, wird das Gewissen aufgrund der Kastrationsangst errichtet. Ihm zufolge entsteht die Kastrationsangst des Jungen aufgrund des Anblicks des weiblichen Geschlechts und derWarnung von Eltern und Pflegepersonen vor den Folgen der Masturbation (vgl. Freud, 1908c, S. 179). Freud war der Ansicht, Frauen hätten keine Kastrationsangst (vgl. Freud, 1926, S. 153). ${ }^{7}$ Judith Le Soldat fand hingegen bei allen Analysandinnen Material, wie Camille es präsentiert. Wie Le Soldat entdeckte, entsteht die Kastrationsangst infolge des in der Kindheit begangenen ödipalen Raubs am Vater - und zwar bei beiden Geschlechtern (Le Soldat, 2015, S. 148). Laut ihr kastrieren wir alle im Innern den Vater, darum leben wir in der ständigen Angst, die Beute werde von uns zurückgefordert. ${ }^{8}$

Camille erzählt von dieser Kastration, die sie in der Fantasie einst selber begangen hat («zu aggressiv»), und fürchtet nun, dass ihr dasselbe droht. Die Vorstellung, ihr werde der Penis abrasiert, trifft dies präzis: Gemeint ist der Phallus, jener seit dem ödipalen Raub am Vater im Innern versteckte Penis. Die «Geschichten, die in mir sind» meinen die ödipale Beute (Le Soldat, 2015, S. 148), verknüpft mit der «Geschichte», wie sie zu diesem Beutestück gelangt ist: «Der Raub des väterlichen Phallus [...] und der lebenslange insgeheime Besitz des fatalen Gutes machen die in der psychischen Innenwelt vollzogene Kastration zu jener Ungeheuerlichkeit, die unaussprechbar wird» (Le Soldat, 2020, S. 248). Auf dem Höhepunkt ihrer Angst hat Camille die Vorstellung, der Mann fordere etwas Ungeheuerliches von ihr: eine Rasur. Dazu schreibt Le Soldat: «Die Haare schneiden, den Bart opfern [...] sind alles Euphemismen und Symbole der Kastration» (Le Soldat, 2020, S. 286). Freud beschrieb Charcot als «im Gesicht rasiert» und liess «Dr. M.» in seinem Irma-Traum «hink[en], [...] am Kinn bartlos» (Le Soldat, 2020, S. 106). ${ }^{9}$ Beide auf WhatsApp erhaltenen Bilder - Gustaves erhobener blutender Finger und der rasierte Penis - gemahnen Camille, wie die Sphinx, «wortlos, allein durch ihre Erscheinung: «Erinnerst du dich? Erkennst du mich?»» (Le 
Soldat, 2020, S. 398). In Camilles Gedankenstrom, der libidinös-sexuell erregt ist, mischen sich grausige Einfälle. Diese Bilder wirken wie Fingerzeige auf ihre Schuld, eine Mahnung an ihre Schandtaten: Einen Penis hast rasiert, den Vater kastriert. Der Ursprung der Kastrationsangst liegt, wie Le Soldat entdeckte, im ödipalen Raub am Vater (Le Soldat, 2015, S. 148). Mit dieser Tat wird der passiv-genitale Wunsch zum Tabu, weil an ihn die sofort aufkommende Angst vor Strafe geknüpft ist und weil die Frustration ebendieses Wunsches in der Kindheit zur Tat führte. Dieser Wunsch ist tabu, nicht weil der andere böse oder verletzend ist - das sind Projektionen der eigenen Aggression -, sondern weil im Psychischen das Talionsprinzip herrscht und man infolge der eigenen Tat Rache fürchten muss. Camilles Angst ist also deshalb so gross, weil sie selbst einst kastriert hat: Ein Mann weckt in ihr stets die Angst vor dem Rächer (vgl. Le Soldat, 2015, S. 149).

Die aus Camilles salopper Formulierung entstandene Verkürzung «der rasierte Penis» mag eine etwas reisserische Zuspitzung sein, jedoch kann die Kastration kaum deutlicher dargestellt werden: Ein begehrter Penis wird «rasiert». Sobald sich das Begehren auf den Phallus richtet, verbindet sich derWunsch mit der Kastrationstat. Mit diesem Konzept, der Kastrationstat, lassen sich die Phänomene, die Camille im Sexuellen und insbesondere in der Beziehung zu Männern umtreiben, präzise verstehen. Bei ihr als Frau zeigen sich Symptome ${ }^{10}$, da in der Fantasie die Beute in derVagina versteckt ist (vgl. Le Soldat, 2015, S. 148). An dem, was Lust bereitet - am Penetriertwerden vom erigierten Penis -, klebt die Erinnerung an den Raub (Rasur) und an die Drohung, dafür teuer bezahlen zu müssen.

Welche Angstabwehren wendet Camille an? Sie wehrt ihre Angst ab, indem sie erstens ihren Wunsch fallen lässt, zweitens die kontraphobische Flucht nach vorn ergreift (sie lässt sich von einem Draufgänger schlecht behandeln, um mir zu beweisen, dass sie keine Angst vor Männern habe), drittens sich den Frauen zuwendet (das Thema Penetration durch einen Mann ist vom Tisch), viertens beruflich stets die aktive Position ${ }^{11}$ innehat. Weil sie an ihre Schandtat (der blutende Finger), ihre Schuld (der rasierte Penis) und die zu erwartende Strafe (die drohende Rasur) gemahnt wird, stellt sie sich fünftens, als sei die Strafe bereits vollzogen worden, als Kastrationsopfer («ruiniert») dar. ${ }^{12}$ Und sechstens erlaubt die erwähnte Reaktionsbildung den Orgasmus nicht - in Umkehr der Tatsache, dass er dereinst so triumphal stattgefunden hat, als Camille gar nicht sanft war. Ihr Gewissen erachtet ihre damalige Untat als etwa Fünfjährige (vgl. Geiger \& Kappeler, 2021), auf dem Höhepunkt der ödipalen Phase, als unverzeihlich. Die drohende «Krise» weist auf das, worauf es hinauslaufen könnte, wenn Camille ihrem Wunsch folgen würde: «Mann», «Kind» und «Abgrund». 


\section{$5 \quad$ Kastrationsangst und Abwehren aufzeigen}

Im Lauf der Analyse hat sich also gezeigt, dass bei Camille die Angst geweckt wird, sobald sie ihren Liebeswunsch an einen Mann richtet. Sie versucht mit dieser Angst umzugehen und setzt die genannten Abwehren ein. Technisch geht es mir als Analytiker darum, ihre Angstabwehrbewegungen zu benennen und zu beschreiben, welchen Ausweg die Psyche sucht. Dabei macht Camille eine Entwicklung durch. Sie gewinnt ein besseres Verständnis davon, dass «die Sache mit den Männern» mit Angst verbunden ist. Indem sie Gustave besuchen geht, wagt sie es, einem Mann näherzukommen. Ihre Angstabwehrbewegungen zeigen sich aber weiterhin. So sagt sie etwa, sie «fühle» sich zwar «schon hetero» und sei «verliebt», doch als Ausweichmanöver bringt sie vor, sie wolle lieber via Samenspende zu einem Kind kommen. Dies benenne ich:

Sie sagen, Sie seien hetero, aber es gibt eine Angst vor etwas [...], was zwischen Ihnen und den Männern steht. In diesen Mann sind Sie verliebt, finden aber, Sie müssten die Finger von ihm lassen. Ihre jetzige Lösung lautet: auf Männer verzichten. Wenn wir aber herausfinden, wovor Sie Angst haben, wird dieser Verzicht vielleicht überflüssig.

Ich zeige Camille auf, dass sie riskiert, auch ihren Wunsch über Bord zu werfen, wo sie doch nur ihre Angst loswerden will. Dabei geht es mir darum, dass sie ihren inneren Konflikt spürt. Im weiteren Analyseverlauf taucht die neue Idee auf, Camille müsse sich einen Mann «schnappen» - worauf sich unmittelbar das Gefühl meldet, dieser würde bestimmt bald wieder nach anderen Frauen Ausschau halten. Ich beschreibe ihr diese Dynamik:

Sie wollen einen Mann, doch Einsprüche werden laut. Sie haben die Vorstellung, dass Sie sich ihn nehmen müssen, doch dann meldet sich das Gefühl, in der Folge strebe er weg. Das schlimme Gefühl, das Sie loshaben möchten, ist die stetige Befürchtung, der andere gehe weg.

In meiner Deutung spreche ich die Kastrationsangst an, die für Camilles Hemmung verantwortlich ist, sich einen Mann zu nehmen. Ein Mann, den man sich «schnappen» muss, nimmt danach innerpsychisch den Platz der ödipalen 
Beute ein, die danach strebt, an ihren Ursprungsort zurückzukehren (vgl. Le Soldat 2015, S. 151).

Wie geht es nun in Camilles Analyse weiter? Weiterhin benenne ich sorgfältig Angstabwehr um Angstabwehr, um Camille darin zu unterstützen, das in ihrem Innern stehende Hindernis zu erforschen und zu verstehen; um ihr zu ermöglichen, ihre Angst zu spüren und auszuhalten. Je besser sie diese spürt, desto mehr Raum kann sie ihrem Wunsch gewähren - was wiederum ihre Angst erhöht. Die Angst ist demnach ein Zeichen dafür, dass sie sich ihrem Wunsch nähert. Erst wenn sich Camille dessen bewusst ist, können wir erforschen, woher ihre Angst stammt. Dann kann der Kampf gegen ihr Gewissen geführt werden, das ihr ihren Wunsch hartnäckig verbietet, ihr die alte «Geschichte» vorhält und nach dem Talionsprinzip mit Vergeltung droht. Wie ich mit Elisabeth Geiger an anderer Stelle dargelegt habe (Suter \& Geiger, 2021), geht es in einer Analyse ganz zentral darum, dass die Analysandin ihre Einstellung gegenüber ihrem Gewissen ändern kann.

Die Macht der Kastrationsangst ist nicht hoch genug einzuschätzen. Abwehren der Angst führen dazu, dass Menschen ihr Liebesobjekt, ihren Kinderwunsch oder ihre Karriere aufgeben. Das Ziel der Analyse besteht meines Erachtens darin, der Kastrationsangst ins Auge zu sehen, sie auszuhalten und zu erkennen, dass diese Angst, die uns so beutelt, keine Realangst ist, sondern eine Drohung unseres Gewissens. Dies gilt nicht nur für Camille und nicht nur für Camille als Frau, sondern ganz grundlegend für Frauen wie auch für Männer. Beide Geschlechter wollen beides (Le Soldat, 2015, S. 146): die Erfüllung des passiv-genitalen Wunsches «nach innen» und des aktiven Wunsches, sich «nach aussen» zu zeigen. Holen wir in der analytischen Arbeit einen solchen Wunsch aus dem Verdrängten, aus dem Unbewussten an die Oberfläche, so kommt uns, wie uns die tägliche Erfahrung lehrt, immer etwas «dazwischen», stets stellt sich (noch) eine Vorstellung in den Weg, vergleichbar der Sphinx, die Ödipus den Weg versperrt. Wir müssen erkennen, dass am Wunsch eine verdrängte Geschichte hängt, von der wir am liebsten nichts wissen wollen. Und je mehr wir die Kastrationsangst loswerden wollen, desto mehr müssen wir zur Kenntnis nehmen: An ihr führt kein Weg vorbei. Anders gesagt kommen wir nicht darum herum, uns selbst als Täterin oder Täter zu sehen. ${ }^{13}$ Das fällt niemandem leicht. 


\section{Literatur}

Despentes, V. (2006). King Kong Theorie. Köln: Kiepenheuer \& Witsch.

Fäh, M. (2021). Judith Le Soldat heute. Theorie und Praxis. Bd. 1: Trieb und Ödipus.

Einführung in das Denken und Werk von Judith Le Soldat. Stuttgart: Frommann-Holzboog.

Fäh, M. (2018). Kolposwunsch, Peniswunsch und Kastrationstat. Aspekte einer Erweiterung der Theorie des Ödipuskomplexes nach Judith Le Soldat. Psyche-Z Psychoanal 72, 1-23.

Freud, S. (1895d). Zur Psychotherapie der Hysterie. GW 1, S. 252-312.

Freud, S. (1900). Die Traumdeutung. GW 2.

Freud, S. (1908). Über infantile Sexualtheorien. GW XII, S. 171-188.

Freud, S. (1919). Das Unheimliche. GW XII, S. 229-268.

Freud, S. (1926). Hemmung, Symptom und Angst. GW XIV, S. 111-205.

Freud, S. (1940a [1938]). Die Psychoanalytische Technik. GW XVII, S. 97-108.

Geiger, E. \& Kappeler, Ch. (2021). Beobachtung von Kindern in der ödipalen Phase. In M. Fäh (Hrsg.), Judith Le Soldat heute. Theorie und Praxis. Bd. 1: Trieb und Ödipus. Einführung in das Denken und Werk von Judith Le Soldat. Stuttgart: Frommann-Holzboog, S. 161-230.

Le Soldat, J. (2020). Raubmord und Verrat: eine Analyse von Freuds Irma-Traum. Kritisch revidierte Neuausgabe von Eine Theorie menschlichen Unglücks (1994). Werkausgabe Bd. 3. Stuttgart: Frommann-Holzboog.

Le Soldat, J. (2015). Grund zur Homosexualität. Werkausgabe Bd. 1. Stuttgart: Frommann-Holzboog.

Le Soldat, J. (1993). Revenons à nos moutons! Irrungen im Übertragungskonflikt.

In B. Grossmann-Garger \&W. Parth (Hrsg.), Heilt die Psychoanalyse?Wien: Orac.

Morgenthaler, F. (2005 [1978]). Technik. Zur Dialektik der Psychoanalytischen Praxis. Giessen: Psychosozial-Verlag.

Riviere, J. (1994 [1927]). Weiblichkeit als Maskerade. In L. Weissberg (Hrsg.), Weiblichkeit als Maskerade (S. 34-47), Frankfurt a. M.: Fischer.

Suter, D. \& Geiger, E. (2021). Oliver - Das Schicksal des passiven Wunsches. In M. Fäh (Hrsg.), Judith Le Soldat heute. Theorie und Praxis. Bd. 1: Trieb und Ödipus. Einführung in das Denken und Werk von Judith Le Soldat. Stuttgart: Frommann-Holzboog, S. 311-359. 


\section{Anmerkungen}

1 Der Analytiker ist zu Beginn ein Hilfs-Ich gegen das Gewissen (vgl. Freud, 1940a).

2 Gemäss Freud liegt dem Psychischen grundsätzlich das Talionsprinzip zugrunde (vgl. 1900, S. 116, und 1919, S. 243).

3 Bei der biblischen Gestalt Samson sind die Haare mit Potenz verbunden (vgl. Bibel, Richter 16,17).

$4 \quad$ Kontraphobisch macht sie eine Progression in die Hammerschlag-Vorstellung (vgl. hierzu Le Soldat, 2015, S. 175) - was bei ihr selten vorkommt.

$5 \quad$ Letzteres erschliesst sich aus weiteren, hier nicht angeführten Sequenzen der Behandlung. Im vorliegenden Beitrag wird gewisses Material verdichtet präsentiert, während anderes präzis, en détail, in der Sukzession dargestellt wird.

$6 \quad$ Dies wird weiter unten noch erklärt.

7 Für eine differenzierte Theoriendarstellung siehe Fäh, 2021, S. 15-51.

8 Auch Joan Riviere beschreibt in «Weiblichkeit als Maskerade» (1927) in einem Fallbeispiel das Kastrieren und die Angst vor dem Vater. Virginie Despentes nimmt in ihrem Essay «King Kong Theorie» wiederum Bezug auf Riviere, die den Fall eines «Zwischentyps von Frau, also heterosexuell, aber männlich» schildere, «die darunter litt, dass sie nach jedem öffentlichen Auftritt von entsetzlicher Angst gepackt wurde, die ihr alle Kraft raubte und sich in einem zwanghaften und demütigenden Drang äusserte, die Aufmerksamkeit der Männer zu suchen» (Despentes, 2006, S. 20 f.). Riviere selbst erläutert: «Durch die Analyse stellte sich dann heraus, dass sich ihr zwanghaftes Flirten und Kokettieren [...] folgendermassen erklärte: Es war der unbewusste Versuch, sich gegen die Angst zurWehr zu setzen, die sich einstellte, weil sie nach der intellektuellen Leistung ihres Vortrags Vergeltungsmassnahmen vonseiten der Vaterfigur befürchtete. Die öffentliche Zurschaustellung ihrer geistigen Fähigkeiten, die sie an sich erfolgreich durchführte, bedeutete, dass sie sich selbst als im Besitz des Penis ihres Vaters zur Schau stellte, nachdem sie ihn kastriert hatte. Sobald dieVorführung vorüber war, wurde sie von einer furchtbaren Angst vor der Vergeltung, die ihr Vater üben würde, erfasst. Offensichtlich war das Bestreben, sich ihm sexuell hinzugeben, ein Versuch, den Rachesuchenden zu besänftigen» (Riviere, 1927, S. 37). - Dass Despentes Rivieres Analysandin als «heterosexuell, aber männlich» bezeichnet, fusst vermutlich auf dem Umstand, dass Freud für die Triebrichtungen «aktiv» und «passiv» unglücklicherweise die Begriffe «männlich» und «weiblich» verwendet hat. Gerade an Camilles Fall lässt sich gut zeigen, dass auch beim passiv-genitalen Wunsch, also beim an den Mann gerichteten Liebeswunsch (den Freud klassisch als «weiblich» bezeichnet hat), mächtige Kastrationsangst aufkommen kann.

9 «Zur symbolischen Darstellung der Kastration dient der Traumarbeit: die Kahlheit, das Haarschneiden, der Zahnausfall und das Köpfen» (Freud, 1900, S. 362).

10 Laut Le Soldat «entsteht die Angst, es würde sich beim Koitus ein heftiger Zusammenstoss in der Vagina zwischen den zwei Körpern ergeben, ein kriegerischer, blutiger Zwischenfall mit eklatanten Folgen, wobei man beide, das innere Objekt, aber auch den Liebhaber verlieren würde. [...] Diese Idee, vielmehr die ihr unterliegende Triebdynamik ist die geläufigste Ursache für die sogenannte «Frigidität` der Frau, und eine der mannigfachen Ursachen männlicher Impotenz. Weil der Mann eben insgeheim auch um das Versteck der Frau weiss, sich zuletzt doch scheut, ‘dort hinein` zu gehen, wo er etwas Lebendiges, Feindseliges erwartet [...]. Dass realiter dort nichts ist, interessiert niemanden, weder Mann noch Frau, denn in der Innenwelt ist es so [Hervorhebung d. Verf.]. Man will aber auch nicht über die Beziehungen von seelischer Realität und physischer Welt aufgeklärt werden, denn die Lust, an den entsprechenden Vorstellungen festzuhalten, mag sie sich im Bewusstsein noch so sehr als Angst darstellen, ist grösser, als es jede Nüchternheit sein könnte» Le Soldat, 2015, S. 191. 
11 Die phallische Hemmung beim aktiven Zeigen dessen, was sie kann, löst sich jeweils schnell wieder.

12 Le Soldat zeigt auf, dass «die Identifikation ein komplexes Abwehrmanöver ist, welche eine im Innern gewichtige Schuld voraussetzt» (Le Soldat, 2015, S. 118).

13 Vgl. Le Soldat, 1993, S. 67.

\section{Angaben zum Autor}

Dominic Suter, 1976, lic. phil., Psychoanalytiker in eigener Praxis in Zürich, Dozent am Psychoanalytischen Seminar Zürich (PSZ) und am Graduate Campus UZH, arbeitete bis 2019 auch an der Psychologischen Beratungsstelle für Studierende und Doktorierende der UZH und der ETH Zürich. dominicsuter.ch 